\title{
Aprendizagem móvel no Brasil: um mapeamento de teses e dissertações
}

Jaqueline Nunes Carvalho aquelinencarv@gmail.com 0000-0002-4863-6847

Universidade do Estado de Mato Grosso, Barra do Bugres, Mato Grosso

Edinéia Aparecida dos Santos Galvanin

galvanin@gmail.com

0000-0002-6678-9599

Universidade do Estado de Mato

Grosso, Barra do Bugres, Mato

Grosso.

Welvesley da Silva Santos welvesleysantos@gmail.com 0000-0002-0630-5564

Universidade do Estado de Mato Grosso, Barra do Bugres, Mato

Grosso.
RESUMO

Esse artigo objetiva mapear as teses e dissertações brasileiras que abordam sobre a aprendizagem móvel nas áreas de educação e/ou ensino. Trata-se de uma pesquisa de caráter documental, que como fonte de dados utilizou o banco de teses e dissertações da CAPES, realizando buscas por palavras-chave com um recorte dos trabalhos publicados a partir de 2013 até novembro de 2016 e área de concentração voltadas para a educação e/ou ensino. As análises realizadas levantaram dados referentes a: distribuição dos trabalhos por ano, titulação, regiões, instituições, área de concentração, linha de pesquisa e tema de estudo. Os resultados indicaram o cenário da aprendizagem móvel no Brasil, e possibilitou verificar as lacunas dessa linha de pesquisa ainda emergente.

PALAVRAS-CHAVE: Aprendizagem móvel. Mapeamento. Educação. Ensino. 


\section{INTRODUÇÃO}

É comum ouvirmos que vivemos em uma 'Era tecnológica' tal significado pode ser atribuído a velocidade com que as tecnologias em especial as digitais vêm evoluindo, e como estão impregnadas no nosso dia-a-dia, possibilitando assim que novos conceitos de aprendizagem se inicie.

$\mathrm{Na}$ busca por otimizar o espaço e o tempo, as tecnologias de mobilidade ganham força e fazem com que a partir dela o conceito de aprendizagem móvel (mobile learning ou m-learnig) venha a emergir.

A aprendizagem móvel se tornou mais conhecida e pesquisada a partir do ano de 2005 , e desde então a busca por definições para o termo, por conhecer suas potencialidades e limitações entre outros aspectos vem sendo objeto de estudo de vários pesquisadores (CROMPTON, 2013).

Existem várias definições para o termo, no entanto, no início à sua maioria era voltada para uma definição mais tecnocêntrica, ou seja, a tecnologia móvel (dispositivos móveis) era vista como a parte principal nesse processo de aprendizagem. Nesse sentido, Borba et al. (2016) cita que a definição do termo não envolve apenas a utilização de recursos tecnológicos, mas sim, a possibilidade de aprender em diferentes contextos.

Crompton (2013) descreve quatro constructos centrais que precisam estar presentes na definição do termo aprendizagem móvel, são eles: a pedagogia, os dispositivos tecnológicos, o contexto e a interação social, e assim sendo, a define como "aprendizagem em vários contextos, por meio de interações sociais e de conteúdo, usando dispositivos eletrônicos pessoais" (ibidem, p. 4). Por contexto a autora refere-se aos espaços formais e informais de aprendizagem.

A aprendizagem móvel é desprovida de restrições espaciais ou temporais, e tem como palavra-chave a mobilidade. Saccol, Schelmmer e Barbosa (2011, p. 65) refere-se à mobilidade em seus diferentes tipos que são:

\footnotetext{
Mobilidade física dos sujeitos (deslocamentos, espaços diferentes); a mobilidade da tecnologia (tipo de dispositivo); a mobilidade conceitual (diferentes conceitos e conteúdos com os quais tenho contato); a mobilidade sociointeracional (interação com diferentes níveis e grupos sociais); e a mobilidade temporal (momentos diferentes).
}

A mobilidade em todos os seus aspectos possibilita para que ocorra a aprendizagem móvel, nesse sentido Traxler (2011) descreve cinco maneiras em que a mesma oferece oportunidade de aprendizagem, são elas: 1) aprendizagem contingente, 2) aprendizagem situada, 3) aprendizagem autêntica, 4) aprendizagem em contexto, 5) aprendizagem personalizada.

Em outras palavras, ela proporciona várias ocasiões de aprendizagem, como: o sendo aluno ativo no processo, a localização como facilitadora de aplicações, a realização de atividades imediatas, a história e o ambiente como parte do processo, além de evidenciar as preferências de acordo com o perfil do aprendiz (TRAXLER, 2011).

Considerando esses aspectos, temos que a aprendizagem móvel possui grande potencial a ser explorado, além disso, conforme Bairral, Assis e Silva (2015) a tecnologia digital tem tomado conta da vida dos jovens, em especial os mais variados modelos de dispositivos móveis já fazem parte do cotidiano, eles são o 
que Prensky (2001) chama de "nativos digitais". Tal situação evidencia a necessidade e as possibilidades de difusão dessa temática.

Desse modo, levando em consideração esse contexto da aprendizagem móvel, esse artigo objetiva mapear teses e dissertações brasileiras disponíveis no banco de teses e dissertações da Coordenação de Aperfeiçoamento de Pessoal de Nível Superior (CAPES) que abordem sobre aprendizagem móvel nas áreas de educação e/ou ensino.

\section{PROCEDIMENTOS METODOLÓGICOS}

Esse artigo se caracteriza como pesquisa documental e para Ferreira (2002, p. 257) as pesquisas desse tipo possuem caráter bibliográfico bem como:

\section{[...] parecem trazer em comum o desafio de mapear e de discutir uma certa produção acadêmica em diferentes campos do conhecimento, tentando responder que aspectos e dimensões que vêm sendo destacados e privilegiados em diferentes épocas e lugares, de que formas e em que condições têm sido produzidas certas dissertações de mestrado, teses de doutorado, publicações em periódicos e comunicações em anais de congressos e de seminários.}

Em outras palavras podemos dizer que esse tipo de pesquisa possibilita compreender como está determinado o conhecimento ao longo do tempo, ou seja, 'o que', 'onde', 'quando' e 'quanto' vem sendo produzido sobre tal assunto.

Retomando desse modo o objetivo desse artigo que é mapear teses e dissertações brasileiras que abordem sobre aprendizagem móvel, esse trabalho permite conhecer um pouco do atual cenário desta área de pesquisa emergente do século XXI.

Assim sendo, para coleta dos dados foi realizado um levantamento no banco de teses e dissertações da CAPES $^{2}$, filtrando os trabalhos que foram publicados no período de 2013 a 2016. A justificativa desse período se faz por dois motivos: primeiro, pelo avanço nas definições de aprendizagem móvel, que em seu início eram bastante tecnicistas (foco no dispositivo e não aprendizagem), e somente a partir de 2013 é que novos constructos são considerados ao se referir ao termo (CROMPTON, 2013); e segundo, a atualidade dos trabalhos já que essa é uma área ainda emergente.

Seguindo a filtragem selecionamos os trabalhos por palavras-chave, que foram: "m-learning", "mobile learning", "aprendizagem móvel" e "aprendizagem com mobilidade". O acesso ao banco de teses e dissertações da CAPES ocorreram nos meses de outubro e novembro de 2016.

Na primeira busca filtramos 72 resultados, no entanto, desses haviam vários que estavam voltados para o desenvolvimento de aplicativos, marketing, administração, engenharia entre outros assuntos, como essas áreas de pesquisa não contemplam e nem respondem ao objetivo desse artigo, realizamos um novo recorte na pesquisa e filtramos os trabalhos que possuíam área de concentração envolvendo educação e/ou ensino, e nesta busca encontramos 25 resultados. 0 Quadro 1, mostra o ano de defesa, autor e título dos trabalhos selecionados. 
Quadro 1 - Informações sobre as teses e dissertações selecionadas - corpus do artigo.

\begin{tabular}{|c|c|c|}
\hline ANO & AUTOR & TÍTULO \\
\hline 2013 & $\begin{array}{l}\text { SOUZA, M. I. } \\
\text { F. }\end{array}$ & $\begin{array}{l}\text { Modelo de produção de microconteúdo educacional para } \\
\text { ambientes virtuais de aprendizagem com mobilidade }\end{array}$ \\
\hline 2013 & $\begin{array}{l}\text { SANTOS, N. S. } \\
\text { R. S. }\end{array}$ & $\begin{array}{l}\text { M-roamin: um modelo para representação de objetos de } \\
\text { aprendizagem multimodais e interativos }\end{array}$ \\
\hline 2014 & $\begin{array}{l}\text { BARROS, M. } \\
\text { A. M. }\end{array}$ & $\begin{array}{l}\text { Concepções, usos, modelos e estratégias da utilização de } \\
\text { dispositivos móveis: uma análise da aprendizagem móvel } \\
\text { entre professores de ciências em formação Recife }\end{array}$ \\
\hline 2014 & $\begin{array}{l}\text { FRANCA, M. } \\
\text { P. }\end{array}$ & $\begin{array}{l}\text { Contribuições dos tablets para as aulas práticas de biologia no } \\
\text { ensino médio }\end{array}$ \\
\hline 2014 & JACON, L. S. C. & $\begin{array}{l}\text { Dispositivos móveis no ensino de Química: o professor } \\
\text { formador, o profissional de informática e os diálogos possíveis }\end{array}$ \\
\hline 2014 & $\begin{array}{l}\text { RODRIGUES, } \\
\text { S.J.S. }\end{array}$ & $\begin{array}{c}\text { English gap: um aplicativo móvel para o ensino de língua } \\
\text { inglesa em escolas públicas }\end{array}$ \\
\hline 2014 & $\begin{array}{l}\text { MOURA, F. A. } \\
\text { D. }\end{array}$ & $\begin{array}{l}\text { O design instrucional de um aplicativo m-learning à educação } \\
\text { matemática: focando o desenvolvimento de atividades- } \\
\text { referentesa-funções-trigonométricas-com-tecnologias-móveis }\end{array}$ \\
\hline 2014 & DIAS, E. J. & O uso dos tablets nas aulas de Matemática no ensino médio \\
\hline 2014 & $\begin{array}{l}\text { SCHNEIDER, } \\
\text { C. K. }\end{array}$ & $\begin{array}{l}\text { Parâmetros visuais como apoio à produção de vídeos } \\
\text { educacionais para o ensino de ciência e tecnologia no } \\
\text { contexto da mobilidade e conectividade }\end{array}$ \\
\hline 2014 & ARAUJO, J. L. & $\begin{array}{l}\begin{array}{l}\text { Segurança pública ostensiva pernambucana conectada por m- } \\
\text { learning' }\end{array} \\
\end{array}$ \\
\hline 2015 & $\begin{array}{l}\text { MORAES, M. } \\
\text { S. }\end{array}$ & $\begin{array}{l}\text { Ambiente de aprendizagem personalizado: proposta para um } \\
\text { sistema baseado em sensibilidade ao contexto }\end{array}$ \\
\hline 2015 & LIMA, M. S. S. & $\begin{array}{l}\text { Aprendizagem colaborativa com apoio computacional: uma } \\
\text { experiência no ensino fundamental }\end{array}$ \\
\hline 2015 & FREITAS, E. A. & $\begin{array}{l}\text { Aprendizagem móvel (m-learning): um estudo acerca da } \\
\text { aplicabilidade de tecnologias móveis na alfabetização de } \\
\text { jovens e adultos }\end{array}$ \\
\hline 2015 & $\begin{array}{l}\text { FERREIRA, D. } \\
\text { F. M. A. }\end{array}$ & $\begin{array}{l}\text { Aprendizagem móvel no ensino superior: o uso do } \\
\text { smartphone por alunos do curso de pedagogia }\end{array}$ \\
\hline 2015 & NEVES, M. S. & $\begin{array}{l}\text { Estratégias para o ensino de gêneros textuais com utilização } \\
\text { de MOOCS em dispositivos móveis' }\end{array}$ \\
\hline 2015 & $\begin{array}{l}\text { FERREIRA, O. } \\
\text { S. }\end{array}$ & História/EAD uma experiência pedagógica m-learning \\
\hline 2015 & $\begin{array}{l}\text { GUAZZELLI, D. } \\
\text { C. H. R. }\end{array}$ & $\begin{array}{l}\text { Inovações pedagógicas com o uso de smartphone com base } \\
\text { no olhar discente }\end{array}$ \\
\hline 2015 & $\begin{array}{l}\text { BERNARDO, J. } \\
\text { C. O. }\end{array}$ & $\begin{array}{l}\text { Leitura em dispositivos móveis digitais na formação inicial de } \\
\text { professores }\end{array}$ \\
\hline 2015 & $\begin{array}{l}\text { SANTANA, J. } \\
\text { A. N. }\end{array}$ & Mobile-learning: uma brincadeira de faz de conta? \\
\hline 2015 & $\begin{array}{l}\text { KULCSAR, M. } \\
\text { C. N. }\end{array}$ & $\begin{array}{l}\text { O ensino de Física com tablets: uma proposta usando mapas } \\
\text { conceituais }\end{array}$ \\
\hline 2015 & LADEIRA, V. P. & $\begin{array}{l}\text { O ensino do conceito de funções em um ambiente } \\
\text { tecnológico: uma investigação qualitativa baseada na teoria } \\
\text { fundamentada sobre a utilização de dispositivos móveis em } \\
\text { sala de aula como instrumentos mediáticos da aprendizagem }\end{array}$ \\
\hline 2015 & LIZ, N. & $\begin{array}{l}\text { Tecnologia móvel no ensino e aprendizagem de língua inglesa } \\
\text { na escola }\end{array}$ \\
\hline
\end{tabular}




\begin{tabular}{ccc}
\hline 2015 & NICHELE, A. G. & $\begin{array}{c}\text { Tecnologias móveis e sem fio nos processos de ensino e de } \\
\text { aprendizagem em Química: uma experiência no instituto } \\
\text { federal de educação, ciência e tecnologia do Rio Grande do } \\
\text { Sul }\end{array}$ \\
\hline 2015 & SOUZA, R. D. & $\begin{array}{c}\text { Uma sequência didática para o ensino da Matemática } \\
\text { probabilística na terceira série do ensino médio com apoio de } \\
\text { dispositivos móveis' }\end{array}$ \\
\hline 2016 & $\begin{array}{c}\text { TEIXEIRA, R. T. } \\
\text { M. }\end{array}$ & $\begin{array}{c}\text { Construção e uso de um aplicativo para android como auxílio } \\
\text { ao ensino de Física }\end{array}$ \\
\hline
\end{tabular}

\section{RESULTADOS E DISCUSSÃO}

Os 25 trabalhos selecionados como corpus desse artigo serão apresentados e discutidos no que se refere a distribuição dos trabalhos por ano, titulação, regiões, instituições, área de concentração, linha de pesquisa e tema de estudo.

Quanto a distribuição de trabalhos por ano, encontramos dois 'picos'3 de produção que ocorreram no ano de 2014 e 2015, como mostra a figura 1.

Figura 1 - Quantidade de produções de teses e dissertações identificadas como corpus desse artigo

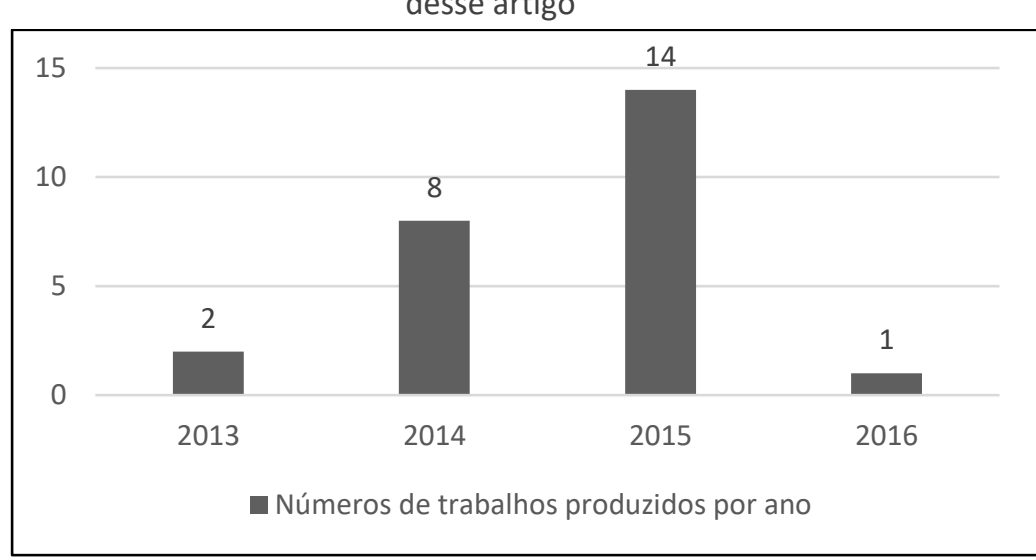

(Fonte: Autores.)

Observando a figura 1 podemos entender que os 'picos' de produção se justificam por essa área de pesquisa ser ainda emergente, conforme salienta Borba et al. (2016) as pesquisas sobre aprendizagem móvel ainda são limitadas, mas está crescendo rapidamente nos últimos anos.

Além desse fator podemos destacar o que diz Almeida e Araújo (2013, p. 30) que a "progressiva diminuição do custo dos dispositivos móveis, maior quantidade de marcas e modelos de tablets no mercado, incentivo a projetos governamentais para uso dos tablets no ensino fundamental e médio" são algumas das razões para o crescente número de pesquisas nessa área.

A respeito do ano de 2016 ainda não apresentar uma quantidade expressiva de trabalhos, esse fator decorre da atualização do banco de teses e dissertações da CAPES, pois, os trabalhos a serem defendidos no corrente ano ainda serão publicados.

Quanto ao tipo de titulação desses trabalhos temos que $80 \%$ desses referemse a mestrados (dissertações) e $20 \%$ a doutorados (teses). Ainda dos $80 \%$ de 
mestrados, $52 \%$ referem-se a mestrados profissionais e $28 \%$ a mestrados acadêmicos.

A quantidade de trabalhos de mestrados nessa área, podem estar associados segundo os dados do Sistema de Informações Georreferenciadas da CAPES (GeoCAPES), a própria quantidade de programas nessa modalidade, sendo que no ano de 2015 há 1810 programas em nível de mestrado e apenas 64 em nível de doutorado.

Nessa circunstância Almeida e Araújo (2013, p. 30) apontam ainda para "a necessidade de intensificação de produção e divulgação de pesquisas, sobretudo teses e dissertações, que abordem sobre o uso de tecnologias móveis na educação formal no Brasil". Vale destacar que são as tecnologias móveis (dispositivos móveis) parte essencial do processo para que ocorra a aprendizagem móvel. Quanto a distribuição por região desses trabalhos temos o que mostra o Quadro 2.

Quadro 2 - Distribuição das dissertações e teses de acordo com as regiões, estados e cidades brasileiras do corpus desse artigo.

\begin{tabular}{|c|c|c|c|c|}
\hline REGIÃO & ESTADO & CIDADE & $\begin{array}{c}\text { № DE } \\
\text { TRABALHOS } \\
\end{array}$ & TOTAL \\
\hline \multirow{5}{*}{ NORDESTE } & BAHIA & SALVADOR & 1 & \multirow{5}{*}{10} \\
\hline & CEARÁ & FORTALEZA & 1 & \\
\hline & PARAÍBA & JOÃO PESSOA & 1 & \\
\hline & PERNAMBUCO & RECIFE & 6 & \\
\hline & $\begin{array}{c}\text { RIO GRANDE DO } \\
\text { NORTE }\end{array}$ & NATAL & 1 & \\
\hline \multirow{6}{*}{ SUDESTE } & \multirow{2}{*}{ MINAS GERAIS } & UBERABA & 1 & \multirow{6}{*}{9} \\
\hline & & OURO PRETO & 1 & \\
\hline & \multirow{4}{*}{ RIO DE JANEIRO } & $\begin{array}{l}\text { DUQUE DE } \\
\text { CAXIAS }\end{array}$ & 1 & \\
\hline & & VASSOURAS & 1 & \\
\hline & & SÃO PAULO & 4 & \\
\hline & & CAMPINAS & 1 & \\
\hline CENTRO-OESTE & MATO GROSSO & CUIÁBA & 1 & 1 \\
\hline \multirow{5}{*}{ SUL } & \multirow[t]{2}{*}{ PARANÁ } & LONDRINA & 1 & \multirow{5}{*}{5} \\
\hline & & PELOTAS & 1 & \\
\hline & \multirow{3}{*}{ RIO GRANDE DO SUL } & PORTO ALEGRE & 1 & \\
\hline & & CANOAS & 1 & \\
\hline & & SÃO LEOPOLDO & 1 & \\
\hline
\end{tabular}

Podemos identificar de acordo com o Quadro 2 que a distribuição por região, estado e cidade do corpus desse artigo está concentrada principalmente nas regiões Nordeste e Sudeste, aparecendo ainda as regiões Centro-Oeste e Sul.

O destaque para a produção desigual pelo território brasileiro pode ser justificado por fatores desde econômicos e políticos até a quantidade de programas nas respectivas regiões. Ramalho $(2005$, p. 75$)$ menciona que: 
Corroborando com Ramalho, Bitar (2010) cita que o alto grau de desenvolvimento e investimento em algumas regiões do país justificam a maior quantidade de publicações acadêmicas, uma vez que esses grandes centros apresentam maior quantidade de Universidades e cursos de pós-graduação strictosensu.

Apesar da região Nordeste não ser considerada um 'grande centro' como as regiões Sudeste e Sul, ela apresenta em especial nos estados que aparecem no Quadro 2 quantidades de programas proporcionais aos da Região Sudeste e Sul (CAPES, 2015).

Quanto a Natureza Administrativa das Instituições que constitui o corpus desse artigo, temos que $60 \%$ dos trabalhos são oriundos de instituições federais, $12 \%$ de instituições estaduais e $28 \%$ de instituições privadas, ou seja, tanto as instituições públicas (federais e estaduais) quanto as privadas contribuíram para essa área de produção acadêmica, conforme descreve o Quadro 3.

Quadro 3 - Natureza Administrativa das Instituições de Ensino do corpus desse artigo.

\begin{tabular}{|c|c|c|c|}
\hline $\begin{array}{c}\text { NATUREZA } \\
\text { ADMINISTRATIVA }\end{array}$ & INSTITUIÇÃO DE ENSINO & $\begin{array}{c}\text { № DE } \\
\text { TRABALHOS }\end{array}$ & TOTAL \\
\hline \multirow{3}{*}{ ESTADUAL } & $\begin{array}{c}\text { UNIVERSIDADE DO ESTADO DA } \\
\text { BAHIA }\end{array}$ & 1 & \multirow{3}{*}{3} \\
\hline & $\begin{array}{l}\text { UNIVERSIDADE DO ESTADO DO } \\
\text { RIO DE JANEIRO }\end{array}$ & 1 & \\
\hline & $\begin{array}{l}\text { UNIVERSIDADE ESTADUAL DE } \\
\text { CAMPINAS }\end{array}$ & 1 & \\
\hline \multirow{11}{*}{ FEDERAL } & $\begin{array}{l}\text { UNIVERSIDADE FEDERAL DA } \\
\text { PARAÍBA/OÃOPFSSOA }\end{array}$ & 1 & \multirow{11}{*}{15} \\
\hline & $\begin{array}{l}\text { UNIVERSIDADE FEDERAL DE } \\
\text { MATO GROSSO }\end{array}$ & 1 & \\
\hline & $\begin{array}{l}\text { UNIVERSIDADE FEDERAL DE } \\
\text { OURO PRETO }\end{array}$ & 1 & \\
\hline & $\begin{array}{l}\text { UNIVERSIDADE FEDERAL DE } \\
\text { PERNAMBUCO }\end{array}$ & 1 & \\
\hline & $\begin{array}{l}\text { UNIVERSIDADE FEDERAL DO } \\
\text { CEARÁ }\end{array}$ & 1 & \\
\hline & $\begin{array}{l}\text { UNIVERSIDADE FEDERAL DO RIO } \\
\text { GRANDE DO SUL }\end{array}$ & 1 & \\
\hline & $\begin{array}{l}\text { UNIVERSIDADE FEDERAL DO } \\
\text { TRIÂNGULO MINEIRO }\end{array}$ & 1 & \\
\hline & $\begin{array}{c}\text { UNIVERSIDADE FEDERAL RURAL } \\
\text { DE PERNAMBUCO }\end{array}$ & 5 & \\
\hline & $\begin{array}{l}\text { UNIVERSIDADE TECNOLÓGICA } \\
\text { FEDERAL DO PARANÁ }\end{array}$ & 1 & \\
\hline & $\begin{array}{l}\text { INSTITUTO FED. DE EDUC, CIÊN. } \\
\text { E TECN. DO RIO GRANDE DO } \\
\text { NORTE }\end{array}$ & 1 & \\
\hline & $\begin{array}{l}\text { INSTITUTO FEDERAL DE EDUC., } \\
\text { CIÊNC. E TECN. SUL-RIO- } \\
\text { GRANDENSE }\end{array}$ & 1 & \\
\hline
\end{tabular}




\begin{tabular}{ccc}
\hline UNIVERSIDADE CRUZEIRO DO & 3 \\
SUL & & \\
UNIVERSIDADE DO VALE DO RIO & 1 & 7 \\
DOS SINOS & 1 & \\
PRIVADA & UNIVERSIDADE LUTERANA DO & 1 \\
BRASIL & 1 \\
UNIVERSIDADE NOVE DE JULHO & 1 \\
UNIVERSIDADE SEVERINO & 1 \\
SOMBRA & \\
\hline
\end{tabular}

As informações que se referem aos programas, áreas de concentração e linhas de pesquisa segundo a classificação da CAPES segue no Quadro 4.

Quadro 4 - Programa, área de concentração e linha de pesquisa do corpus desse artigo

\begin{tabular}{|c|c|c|c|}
\hline PROGRAMA & $\begin{array}{c}\text { ÁREA DE } \\
\text { CONCENTRAÇÃO }\end{array}$ & LINHA DE PESQUISA & $\begin{array}{c}\text { NNo DE } \\
\text { TRABALHOS }\end{array}$ \\
\hline \multirow{7}{*}{ EDUCAÇÃO } & \multirow{6}{*}{ EDUCAÇÃO } & $\begin{array}{c}\text { EDUCAÇÃO, } \\
\text { DESENVOLVIMENTO } \\
\text { E TECNOLOGIAS } \\
\end{array}$ & 1 \\
\hline & & FUNDAMENTOS E & \\
\hline & & PRÁTICAS & 1 \\
\hline & & EDUCACIONAIS & \\
\hline & & $\begin{array}{c}\text { CIÊNCIAS SOCIAIS NA } \\
\text { EDUCAÇÃO }\end{array}$ & 1 \\
\hline & & EDUCAÇÃO POPULAR & 1 \\
\hline & EDUCACAO BRASILEIRA & $\begin{array}{c}\text { EDUCAÇÃO, } \\
\text { CURRÍCULO E ENSINO }\end{array}$ & 1 \\
\hline $\begin{array}{c}\text { EDUCAÇÃO E } \\
\text { CONTEMPORANEIDADE }\end{array}$ & $\begin{array}{c}\text { EDUCAÇÃO E } \\
\text { CONTEMPORANEIDADE }\end{array}$ & $\begin{array}{c}\text { PROCESSOS } \\
\text { CIVILIZATÓRIOS: } \\
\text { EDUCAÇÃO, } \\
\text { MEMÓRIA E } \\
\text { PLURALIDADE } \\
\text { CULTURAL }\end{array}$ & 1 \\
\hline \multirow[b]{2}{*}{$\begin{array}{c}\text { EDUCAÇÃO EM CIÊNCIAS } \\
\text { E }\end{array}$} & \multirow[b]{2}{*}{$\begin{array}{c}\text { EDUCAÇÃO EM CIÊNCIAS } \\
\text { E MATEMÁTICA }\end{array}$} & $\begin{array}{c}\text { LINGUAGENS VERBO- } \\
\text { VISUAIS E } \\
\text { TECNOLOGIAS } \\
\end{array}$ & 1 \\
\hline & & $\begin{array}{c}\text { FORMAÇÃO DE } \\
\text { PROFESSORES PARA } \\
\text { A EDUCAÇÃO EM } \\
\text { CIÊNCIAS E } \\
\text { MATEMÁTICA } \\
\end{array}$ & 1 \\
\hline \multirow[b]{2}{*}{$\begin{array}{l}\text { EDUCAÇÃO } \\
\text { MATEMÁTICA }\end{array}$} & \multirow[b]{2}{*}{$\begin{array}{l}\text { EDUCAÇÃO } \\
\text { MATEMÁTICA }\end{array}$} & *NÃO MENCIONADO & 1 \\
\hline & & $\begin{array}{c}\text { EDUCAÇÃO } \\
\text { MATEMÁTICA } \\
\text { SUPERIOR, } \\
\text { INFORMÁTICA } \\
\text { EDUCACIONAL E } \\
\text { MODELAGEM } \\
\text { MATEMÁTICA } \\
\end{array}$ & 1 \\
\hline $\begin{array}{c}\text { EDUCAÇÃO, CULTURA E } \\
\text { COMUNICAÇÃO }\end{array}$ & $\begin{array}{c}\text { EDUCAÇÃO, CULTURA E } \\
\text { COMUNICAÇÃO EM } \\
\text { PERIFERIAS URBANAS }\end{array}$ & $\begin{array}{c}\text { EDUCAÇÃO, ESCOLA } \\
\text { E SEUS SUJEITOS } \\
\text { SOCIAIS }\end{array}$ & 1 \\
\hline
\end{tabular}




\begin{tabular}{|c|c|c|c|}
\hline $\begin{array}{c}\text { EDUCAÇÃO } \\
\text { MATEMÁTICA E } \\
\text { TECNOLÓGICA }\end{array}$ & $\begin{array}{c}\text { ENSINO DE CIENCIAS E } \\
\text { MATEMATICA }\end{array}$ & $\begin{array}{l}\text { EDUCAÇÃO } \\
\text { TECNOLÓGICA }\end{array}$ & 1 \\
\hline ENSINO DAS CIÊNCIAS & $\begin{array}{c}\text { ENSINO DE CIÊNCIAS E } \\
\text { MATEMÁTICA }\end{array}$ & $\begin{array}{c}\text { FORMAÇÃO DE } \\
\text { PROFESSORES E } \\
\text { CONSTRUÇÃO DE } \\
\text { PRÁTICAS DOCENTES } \\
\text { NO ENSINO DE } \\
\text { CIÊNCIAS E } \\
\text { MATEMÁTICA } \\
\end{array}$ & 1 \\
\hline $\begin{array}{c}\text { ENSINO DE CIÊNCIAS E } \\
\text { MATEMÁTICA }\end{array}$ & $\begin{array}{c}\text { ENSINO DE CIÊNCIAS E } \\
\text { MATEMÁTICA }\end{array}$ & $\begin{array}{c}\text { TECNOLOGIAS } \\
\text { COMPUTACIONAIS } \\
\text { APLICADAS AO } \\
\text { ENSINO DE CIÊNCIAS } \\
\text { E MATEMÁTICA } \\
\text { TECNOLOGIAS DE } \\
\text { INFORMAÇÃO E } \\
\text { COMUNICAÇÃO PARA } \\
\text { O ENSINO DE } \\
\text { CIÊNCIAS E } \\
\text { MATEMÁTICA (TIC) }\end{array}$ & 3 \\
\hline $\begin{array}{c}\text { ENSINO DE CIÊNCIAS } \\
\text { HUMANAS, SOCIAIS E DA } \\
\text { NATUREZA }\end{array}$ & $\begin{array}{l}\text { ENSINO, CIÊNCIAS E } \\
\text { NOVAS TECNOLOGIAS }\end{array}$ & $\begin{array}{c}\text { FUNDAMENTOS E } \\
\text { METODOLOGIAS } \\
\text { PARA O ENSINO DE } \\
\text { CIÊNCIAS HUMANAS }\end{array}$ & 1 \\
\hline $\begin{array}{c}\text { ENSINO DE FÍSICA - } \\
\text { PROFIS } \\
\end{array}$ & ENSINO DE FÍSICA & $\begin{array}{c}\text { FÍSICA NO ENSINO } \\
\text { MÉDIO }\end{array}$ & 1 \\
\hline $\begin{array}{c}\text { GESTÃO E PRÁTICAS } \\
\text { EDUCACIONAIS }\end{array}$ & $\begin{array}{c}\text { GESTÃO E PRÁTICAS } \\
\text { EDUCACIONAIS }\end{array}$ & $\begin{array}{l}\text { LINHA DE PESQUISA E } \\
\text { DE INTERVENÇÃO } \\
\text { GESTÃO } \\
\text { EDUCACIONAL } \\
\text { (LIPIGES) }\end{array}$ & 1 \\
\hline $\begin{array}{l}\text { INFORMÁTICA NA } \\
\text { EDUCAÇÃO }\end{array}$ & $\begin{array}{c}\text { AMBIENTES } \\
\text { INFORMATIZADOS E } \\
\text { ENSINO A DISTANCIA }\end{array}$ & $\begin{array}{c}\text { AMBIENTES } \\
\text { INFORMATIZADOS E } \\
\text { ENSINO A DISTÂNCIA } \\
\end{array}$ & 1 \\
\hline $\begin{array}{c}\text { TECNOLOGIA E GESTÃO } \\
\text { EM EDUCAÇÃO A } \\
\text { DISTÂNCIA }\end{array}$ & $\begin{array}{c}\text { TECNOLOGIA E GESTÃO } \\
\text { EM EDUCAÇÃO A } \\
\text { DISTÂNCIA }\end{array}$ & $\begin{array}{c}\text { FERRAMENTAS } \\
\text { TECNOLÓGICAS PARA } \\
\text { EDUCAÇÃO A } \\
\text { DISTÂNCIA }\end{array}$ & 4 \\
\hline
\end{tabular}

Quanto aos dados dos programas descritos no Quadro 4 temos que a maior parte dos trabalhos estão concentrados nos programas de Educação, Ensino de Ciências e Matemática e Tecnologia e Gestão em Educação a Distância. As linhas de pesquisa que possuem mais quantidade de trabalho são "Tecnologias computacionais aplicadas ao ensino de ciências e matemática" e "Ferramentas tecnológicas para educação a distância".

Nessa perspectiva temos alguns fatores que justificam tal comportamento. Como é o caso do objetivo do vigente Plano Nacional da Educação Básica que visa capacitar profissionais para a Educação Básica e para isso dispõe como medida qualificar profissionais em Pós-graduação stricto-sensu na área de Ensino (BRASIL, 2011), unindo a isso, temos os 'nativos digitais' (PRENSKY, 2001) que são o público das nossas escolas, logo, temos a justificativa de que é preciso pesquisar assuntos emergentes que vá ao encontro dessa nova realidade da sociedade. 
Para complementar as áreas de concentração e linhas de pesquisas que constituem o corpus da pesquisa, o Quadro 5 sintetiza os principais temas abordados nesses trabalhos. Aqui nomeamos de 'tema de estudo' o tema central abordado na pesquisa e 'assunto' o objetivo da pesquisa.

Quadro 5 - Tema de Estudo e Assunto do corpus desse artigo.

TEMA DE ESTUDO

\begin{tabular}{|c|c|}
\hline ALFABETIZAÇÃO & $\begin{array}{c}\text { INDICAR TENDÊNCIAS E PROPOSTAS } \\
\text { ENVOLVENDO TECNOLOGIAS MÓVEIS PARA } \\
\text { JOVENS E ADULTOS }\end{array}$ \\
\hline APRENDIZAGEM COLABORATIVA & APOIO DOS DISPOSITIVOS MÓVEIS \\
\hline BIOLOGIA & CONTRIBUIÇÕES DOS TABLETS \\
\hline FícICA & O ENSINO COM TABLETS \\
\hline השונותו & CONTRUÇÃO E USO DE APLICATIVO \\
\hline FORMAÇÃO DE PROFESSORES & $\begin{array}{c}\text { USOS, CONCEPÇÕES, MODELOS E ESTRATÉGIAS } \\
\text { BASEADO NA APRENDIZAGEM MÓVEL } \\
\text { INOVAÇÕES PEDAGÓGICAS COM O USO DE } \\
\text { DIPOSITIVOS MÓVEIS }\end{array}$ \\
\hline FORMAÇÃO DE TUTORES & $\begin{array}{c}\text { PROPOSTA DE SISTEMA DE APRENDIZAGEM } \\
\text { PERSONALIZADO }\end{array}$ \\
\hline HISTÓRIA & ANALISAR UM CONJUNTO DE SOLUÇÕES \\
\hline INGLÊS & $\begin{array}{c}\text { PRODUÇÃO DE APLICATIVO PARA TABLET E } \\
\text { SMARTPHONE } \\
\text { DESENVOLVIMENTO DE APLICATIVO }\end{array}$ \\
\hline LETRAS & $\begin{array}{l}\text { INVESTIGAR A VIABILIDADE DA LEITURA EM } \\
\text { DISPOSITIVOS MÓVEIS }\end{array}$ \\
\hline MATEMÁTICA & $\begin{array}{c}\text { O USO DE TABLETS NO ENSINO } \\
\text { INVESTIGAR AS ESTRATÉGIAS DE } \\
\text { DESENVOLVIMENTO DE UM APLICATIVO M- } \\
\text { LEARNING } \\
\text { SEQUÊNCIA DIDÁTICA COM DISPOSITIVOS } \\
\text { MÓVEIS } \\
\text { CONTRIBUIÇÕES DOS DISPOSITOS MÓVEIS }\end{array}$ \\
\hline M-LEARNING & $\begin{array}{c}\text { SEGURANÇA PÚBLICA } \\
\text { INVESTIGAÇÃO } \\
\text { APRESENTAÇÃO DE UM MODELO DE PRODUÇÃO } \\
\text { DE MICROCONTEÚDO }\end{array}$ \\
\hline OBJETOS DE APRENDIZAGEM & MODELO DE REPRESENTAÇÃO M-ROAMIN \\
\hline PEDAGOGIA & MAPEAR O USO DE SMARTPHONE \\
\hline PORTUGUÊS & POSSIBILIDADES E INOVAÇÃO DE UM APLICATIVO \\
\hline PRODUÇÃO DE VÍDEOS & $\begin{array}{l}\text { ANÁLISE DOCUMENTAL PARA ESTABELECIMENTO } \\
\text { DE PARÂMETROS }\end{array}$ \\
\hline QUÍMICA & $\begin{array}{c}\text { INVESTIGAÇÃO AS CONTRIBUIÇÕES DAS } \\
\text { TECNOLOGIAS MÓVEIS E SEM FIO NO PROCESSO } \\
\text { DE ENSINO APRENDIZAGEM } \\
\text { UTILIZAÇÃO DE DISPOSITIVOS MÓVEIS }\end{array}$ \\
\hline
\end{tabular}

Os trabalhos conforme indicam o Quadro 5 abrangem diversos 'temas de estudo' que vão desde as áreas curriculares até especificidades da 'aprendizagem móvel'. Os assuntos também possuem vários focos que transitam entre testar a utilização de dispositivos móveis até a criação de aplicativos e softwares. 
Concernente a isso, Borba et al. (2016) descreve em uma revisão da literatura realizada sobre aprendizagem móvel que a maior parte dos trabalhos desse tema estão organizados em três categorias, são elas: estudos sobre o potencial dos dispositivos para o ensino e aprendizagem, estudos sobre o efetivo uso do dispositivo e uso do dispositivo na formação de professores. O autor neste trabalho possuía como objetivo descrever as categorias de aprendizagem móvel de matemática, no entanto, podemos considerar que nesse artigo esse cenário de temas de pesquisa se repete.

Nessa perspectiva, a Organização das Nações Unidas para a Educação, a ciência e a cultura (UNESCO) (2014, p. 14) menciona ainda que:

É importante que a implementação de projetos de aprendizagem móvel e seus modelos pedagógicos não sejam orientados apenas pelas vantagens e limitações das tecnologias móveis, mas também pela consciência de como as tecnologias se encaixam na estrutura cultural e social mais ampla das comunidades.

Logo, consideramos que esse artigo possa contribuir para identificação das lacunas que estão ficando nas pesquisas sobre aprendizagem móvel, e quais os caminhos já percorridos para que se efetive essa área de estudo.

\section{CONSIDERAÇÕES FINAIS}

A pesquisa documental por meio do mapeamento possibilitou uma compreensão sobre 'o que', 'onde', 'quando' e 'quanto' vem sendo feito a respeito de determinada área do conhecimento. Desse modo considerando o objetivo desse artigo em mapear as teses e dissertações brasileiras disponíveis no banco de teses e dissertações da CAPES, no período de 2013 a 2016 que abordem sobre aprendizagem móvel nas áreas de educação e/ou ensino, temos uma possibilidade de conhecer desde os dados institucionais até a temática dos mesmos.

A filtragem dos dados desse mapeamento indica que, há uma grande variedade de trabalhos que abordam sobre 'aprendizagem móvel' e por isso foi necessário estabelecer critérios para direcionar a pesquisa visando responder o objetivo da mesma.

Dos trabalhos que constituem o corpus desse artigo, observamos que existem 'picos' de produção nos anos de 2014 e 2015, sendo possível justificar este fator pelo aumento de interesse nessa área que é considerada emergente do século XXI, além das facilidades em obtenção dos dispositivos móveis.

Há também uma diferença considerável na quantidade de trabalhos de mestrado e os de doutorado, fator esse que pode ser atribuído a quantidade de programas existentes no território brasileiro, sendo que existe maior quantidade de mestrados que doutorados.

No que se refere a distribuição geográfica, temos uma maior quantidade de trabalhos publicados nos grandes centros, e regiões mais desenvolvidas do país, reforçando o que a literatura já afirma sobre a desigualdade na distribuição de programas de pós-graduação. 
Quanto as instituições de ensino, tanto as públicas (federal e estadual) quanto as privadas, tem contribuído para o desenvolvimento dessa linha de pesquisa nos últimos anos.

Identificamos várias áreas de concentração e linhas de pesquisas, porém as que apresentaram maior quantidade de trabalhos foram as de 'ensino de ciências e matemática', 'educação' e 'tecnologia e gestão em educação a distância'. Indicando o interesse em desenvolver metodologias e conhecer técnicas novas para aprimoramento de tais áreas.

E por fim, os 'temas de estudo' e os 'assuntos' evidenciaram múltiplos tópicos que estão sendo explorados na aprendizagem móvel. Os temas envolvem não apenas as áreas curriculares (disciplinas básicas), mas também indicam o interesse em investigar sobre as peculiaridades de como funciona e se utiliza a aprendizagem móvel. Os assuntos, vão desde a experimentação da aprendizagem móvel até o desenvolvimento de aplicativos para promoção dessa aprendizagem.

Dessa maneira, consideramos que essa área de pesquisa ainda que emergente está sendo objeto de estudo de vários pesquisadores, no entanto, há muito a ser explorado, desde suas potencialidades e limitações até seus reflexos na sociedade, de modo que preencha as lacunas e peculiaridades da área que ainda merecem atenção. 


\title{
Mobile learning in Brazil: a mapping of theses and dissertations
}

\begin{abstract}
This article aims to map the Brazilian theses and dissertations covering about mobile learning in the areas of education and/or teaching. This is a documentary character, who as the data source used the Bank of theses and dissertations of CAPES, performing keyword searches with a clipping of published works from 2013 until November 2016 and concentration area focused on education and/or teaching. The analysis raised data: distribution of jobs a year, titling, regions, institutions, area of concentration, research and subject of study. The results indicated the mobile learning scenario in Brazil, and made it possible to check the gaps of this line of research still emerging.
\end{abstract}

KEYWORDS: Mobile learning. Mapping. Education. Teaching. 
1 Por 'Nativos Digitais' ele se refere aqueles que já nasceram em uma época em que as tecnologias digitais estavam amplamente difundidas.

2 http://bancodeteses.capes.gov.br/banco-teses/\#/

3 Por 'picos', nesse contexto, entendemos o(s) ponto(s) altos da imagem/gráfico que indicam maior quantidade de produção.

\section{REFERÊNCIAS}

ALMEIDA, R. R.; ARAÚJO, C. A. F. J. O uso de dispositivos móveis no contexto educativo: análise de teses e dissertações nacionais. Revista tempos e espaços em educação, v.6, n. 11, 2013.

BAIRRAL, M. A.; ASSIS, A. R.; SILVA, B. C. C. Uma matemática na ponta dos dedos com dispositivos touchscreen. RBECT, v. 8, n. 4, 2015.

BITAR, Anderson Lulu. Pesquisa em educação Ambiental: A atividade de campo em teses e dissertações. 2010. 170 f. Dissertação (Mestrado em Educação) Programa de Pós-Graduação e Educação, Universidade Estadual Paulista "Júlio de Mesquita Filho", Rio Claro, 2010.

BORBA, M. C.; ASKAR, P.; ENGELBRECHT, J. GADANIDIS, G.; LLINARES, S.; AGUILAR, M. S. Blended learning, e-learning and mobile in mathematics education. ZDM Mathematics Education, 2016.

BRASIL. Ministério da Educação. Coordenação de Aperfeiçoamento de Pessoal de Nível Superior. Plano Nacional de Pós-Graduação -PNPG 2011-2020. Brasília, DF:2010.

CAPES. Coordenação de aperfeiçoamento de pessoal de nível superior. GeoCAPES: visão geográfica e visão analítica. Disponível em: <geocapes.capes.gov.br/geocapes2/>. Acesso em: nov. 2016

Crompton, H. A historical overview of mobile learning: toward learner-centered education. In Z. L. Berge \& L. Y. Muilenburg (Eds.), Handbook of mobile learning (pp. 3-14). Florence: Routledge, 2013. Arte". Educação \& Sociedade, ano XXIII, n. 79, p. 257-272, 2002. Disponível em: $<$ http://www.propp.ufms.br/ppgedu/geppe/textoestadodaarte.pdf $>$. Acesso em nov 2016. 
PRESNKY, M. Digital Natives, Digital Immigrants. On the Horizon. MCB University Press, v.9, n. 5, out. 2001.

RAMALHO, Betania Leite. MADEIRA, Vicente de Paulo Carvalho. A pós-graduação em educação no Norte e Nordeste: desafios, avanços e perspectivas. Revista Brasileira de Educação. n.30, p.70-81, 2005.

SACCOL, A.; SCHLEMMER, E.; BARBOSA, J. M-learning e u-learning: novas perspectivas das aprendizagens móvel e ubíqua. São Paulo: Prentice Hall, 2011.

TRAXLER, J.; WISHART, J. (Org) Making Mobile learning work: case studies of practice. The Higher Education Academy. Subject Centre for Education: Bristol, 2011.

UNESCO, Organização das Nações Unidas para a Educação, Ciência e Cultura. 0 Futuro da aprendizagem móvel: implicações para planejadores e gestores de políticas. Brasília: 2014.

\section{Recebido: 2017-01-26 \\ Aprovado: 2017-04-05 \\ DOI: $10.3895 /$ rbect.v11n1.5364}

Como citar: CARVALHO, J. N.; GALVANIN, E. A. S.; SANTOS, W. S. Aprendizagem móvel no Brasil: um mapeamento de teses e dissertações. Revista Brasileira de Ensino de Ciência e Tecnologia, v. 11, n. 1, 2018. Disponível em: <https://periodicos.utfpr.edu.br/rbect/article/view/5364>. Acesso em: xxx. Correspondência: Jaqueline Nunes Carvalho - jaquelinencarv@gmail.com Direito autoral: Este artigo está licenciado sob os termos da Licença Creative Commons-Atribuição 4.0 Internacional. 